\title{
Detection of coatings within liquid-filled tubes and containers by mode conversion of leaky Lamb waves
}

\author{
M. Schmitt ${ }^{1}$, K. Schmidt ${ }^{1}$, S. Olfert ${ }^{2}$, J. Rautenberg ${ }^{2}$, G. Lindner ${ }^{1}$, B. Henning ${ }^{2}$, and L. M. Reindl ${ }^{3}$ \\ ${ }^{1}$ Coburg University of Applied Sciences, Institute of Sensor and Actuator Technology, \\ Am Hofbrauhaus 1b, 96450 Coburg, Germany \\ ${ }^{2}$ University Paderborn, Faculty of Electrical Engineering, Computer Science and Mathematics, Measurement \\ Engineering Group, Warburger Str. 100, 33098 Paderborn, Germany \\ ${ }^{3}$ University Freiburg, Department of Microsystems Engineering - IMTEK, Laboratory for Electrical \\ Instrumentation, Georges-Koehler-Allee 106, 79110 Freiburg, Germany
}

Correspondence to: M. Schmitt (martin.schmitt@isat-coburg.de)

Received: 18 December 2012 - Accepted: 24 April 2013 - Published: 23 May 2013

\begin{abstract}
In this paper, a new acoustic sensor principle for coating detection within liquid-filled tubes and containers based on mode conversion of leaky Lamb waves is introduced. Leaky Lamb waves are excited and detected by single-phase transducers, which are attached on the outer side of a tube or container. By transmission time and amplitude measurements, coating formation within the liquid-filled tube and container is detected non-invasively. This new sensor principle is subdivided into the separate considerations of Lamb wave excitation, mode conversion and inverse mode conversion. The Lamb wave excitation by a single-phase transducer is visualized by scanning laser Doppler vibrometer imaging. The mode conversion process of leaky Lamb waves is measured by membrane hydrophone measurements and Schlieren visualization; afterwards, the measured emission angles are compared with the theoretical one. The inverse mode conversion process of pressure waves back to leaky Lamb waves is visualized by Schlieren images. By merging the results of Lamb wave excitation, mode conversion and inverse mode conversion, the new sensor concept is explained. Theoretical considerations and measurement results of adhesive tape coating inside a liquid-filled plastic tube and a liquid-filled stainless steel container verify the new acoustic sensor principle. Finally the measuring sensitivity and the technical realization are discussed.
\end{abstract}

\section{Introduction}

The detection of coatings within liquid-filled tubes and containers is relevant for many technical facilities. The application field ranges from water-supplying systems and the food industry to the chemical industry. Different kinds of sensors such as fiber optical sensors, electrochemical sensors, pressure sensors or heat sensors were investigated for the measurement of the presence and the thickness of organic or inorganic deposits (Withers, 1996; Flemming, 2003). Unfortunately, all aforementioned sensors must be brought in direct contact to the coating or the fluid. In contrast, acoustical sensors have the possibility to work non-invasively (Janknecht and Melo, 2003). However, standard ultrasonic sensors driven by the pulse-echo technique fail for soft coating or deposition detection, such as fouling layers, or have a too-low detection limit in the case of the transmission technique (Janknecht and Melo, 2003). Alternatively, guided waves called Lamb waves can be utilized for soft coating or deposition detection (Lohr and Rose, 2003). In this case, nonleaky Lamb wave modes on plates, non-leaky circumferential guided waves on pipes or longitudinal guided waves traveling along the length of the pipe were excited. In all cases, the attenuation of the guided wave caused by the excitation of pressure waves into the adjacent liquid was minimized by choosing the right guided wave mode and excitation frequency (Lohr and Rose, 2003). 

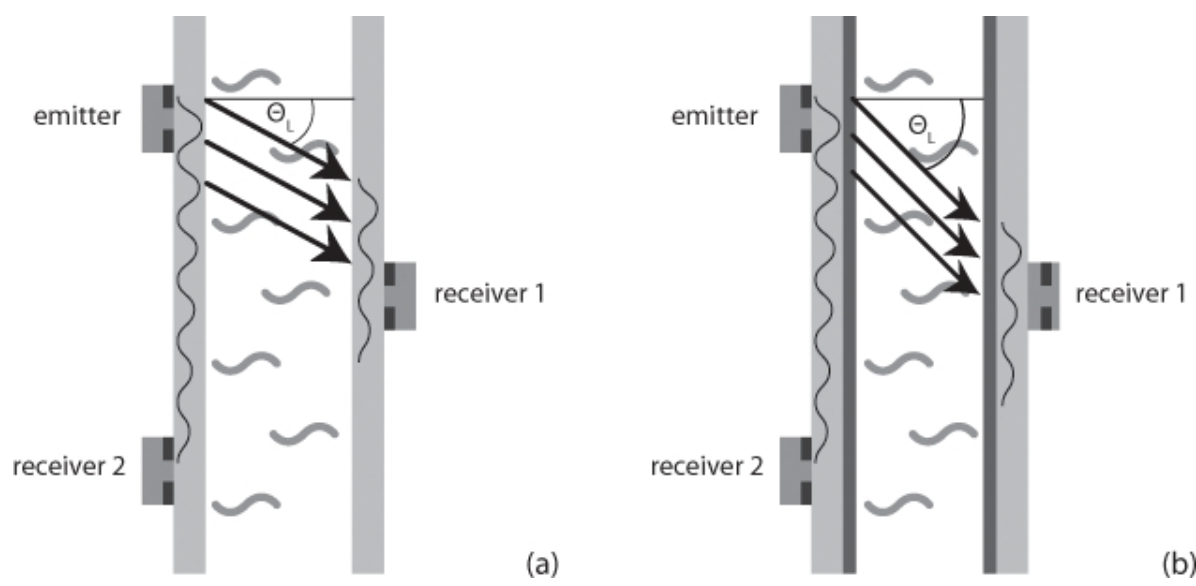

Figure 1. (a) The measurement setup for the new acoustic sensor principle consists of one emitter and two receivers attached to the outer side of a tube or a container. (b) A formation of a coating inside the tube or container results in changes of the transmission times and the amplitudes of the acoustic signals on receiver 1 and receiver 2.

In this paper, a new acoustical sensor concept based on leaky Lamb waves traveling on the wall of tubes or containers is introduced. Leaky Lamb waves, which have a dominant emission into the adjacent liquid, are excited and detected by single-phase transducers, which are attached to the outer side of the tube or the container structure. This means that no sensor element must be inserted into the tube or container. Hence, the boundary of sterility stays intact.

In contrast to former publications in the literature, a leaky Lamb wave on technical common non-piezoelectric substrates, such as steel-, aluminum- or fiber-reinforced plastics is investigated for coating detection. Before, only non-leaky Lamb wave (Lohr and Rose, 2003) on non-piezoelectric substrates or shear horizontal waves on piezoelectric substrates (Balantine et al., 1997; Thomson and Stone, 1997) were investigated, because it was thought that bulk acoustic waves or surface acoustic waves with a dominant vertical displacement would be inapplicable for coating detection in a liquid environment (Länge et al., 2008; Calabrese et al., 1987).

In this journal paper, the new acoustical sensor concept based on mode conversion of Lamb waves will be introduced. Scanning laser Doppler vibrometer images of Lamb wave excitation by single-phase transducers are recorded and compared with diffraction theory. If the excited Lamb wave is in contact with water, mode conversion occurs. The emission angle is measured by a membrane hydrophone measurement and by Schlieren imaging. The evaluated emission angle is compared with the theoretical one. The inverse mode conversion of the mode-converted pressure wave back to a Lamb wave on the opposite side of a two parallel plate arrangement is visualized by Schlieren images. Theoretical considerations and measurement results of coating detection within a plastic tube and a stainless steel container are presented. Finally, the measuring sensitivity and the technical realization of the new acoustical sensor concept will be discussed.

\section{New measurement concept}

The new acoustic measurement principle for coating detection is based on the mode conversion process of Lamb waves. Lamb waves are excited by an emitter attached to the outer side of the tube or container (Fig. 1a). Because the phase velocity of the dominant excited antisymmetrical zero-order Lamb wave is higher than the sound velocity in water, mode conversion occurs. This means that a pressure wave is radiated under a characteristic angle, called Lamb angle, into the adjacent liquid (Fig. 1a). On the opposite side of the tube or container, the pressure wave in the liquid generates a Lamb wave again (Fig. 1a). With receiver 1 on the opposite wall of the tube or container, the sound wave through the liquid can be detected. If the distance between the emitter and receiver 2 on the same side of the tube or container is not too long, the residual Lamb wave can be evaluated too. By burst excitation of the emitter, one part of the wave group travels through the liquid by mode conversion and inverse mode conversion, and the residual part of the wave group remains on the emitter side. Both signals are evaluated for the measurement of the transmission time and the amplitude.

By the formation of a coating inside the tube, the boundary conditions of Lamb wave propagation are changed, resulting in alterations of the radiation angle, the mode conversion and the inverse mode conversion process. These affect the measured transmission times and the amplitudes of the acoustic signals on receiver 1 and receiver 2 . 


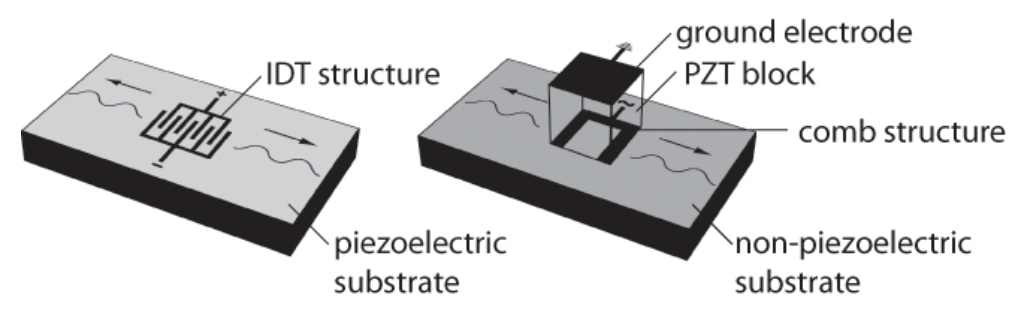

(a)

(b)

Figure 2. (a) Interdigital transducer on a piezoelectric substrate. (b) Single-phase transducer on a non-piezoelectric substrate.

\section{Lamb wave excitation}

Typically, surface acoustic waves are excited on piezoelectric substrates by interdigital transducers (Fig. 2a) (White, 1970). In order to excite surface acoustic waves on non-piezoelectric substrates, a single-phase transducer can be utilized (White, 1970; Lindner, 2008). The single-phase transducer consists of a piezoelectric PZT block, which is metalized with a comb pattern on one side (Fig. 2b). The opposite side of the piezoelectric block is completely metalized (Fig. 2b). On the comb pattern, an electrical high-voltage signal is applied, and on the opposite complete metalized side the electrical ground signal is applied (Fig. 2b). By the right choice of the distance between the fingers of the comb structure, the operation frequency of the transducer can be adjusted. In this paper, a single-phase transducer with a center frequency of $1 \mathrm{MHz}$ is utilized. By gluing the single-phase transducer onto the non-piezoelectric substrates, either Rayleigh or Lamb waves can be excited. If the thickness of the substrate is smaller than the acoustic wavelength, Lamb waves are excited on the substrate; when vice versa, Rayleigh waves are excited (Viktorov, 1967). During the excitation, the deformation of the single-phase transducer is transferred onto the non-piezoelectric substrate. In this way, the pitch of the fingers defines the acoustic wavelength of the excited Lamb or Rayleigh wave.

The phase velocity of Lamb waves depends on the excitation frequency and the plate thickness (Viktorov, 1967). The dispersion curve of a glass plate in Fig. 3 shows the dependency of the phase velocity on the product of frequency and plate thickness. The dispersion curve was calculated by the global matrix method (Lowe, 1995). The basic model for the calculation consists of a thin glass plate with a thickness of $1 \mathrm{~mm}$ having contact to a liquid half-space of water on one side and contact to a vacuum half-space on the other side. In the low frequency thickness region, the antisymmetrical zero-order Lamb wave mode (A0), the symmetrical zero-order Lamb wave mode (SO) and the quasi-Scholte plate mode (QSP) can exist (Fig. 3).

Because the used single-phase transducer has only two fingers with a finite width and the excitation signal is a sinusoidal burst, all modes within an excitation region are excited by the single-phase transducer (Monkhouse et al., 1997)

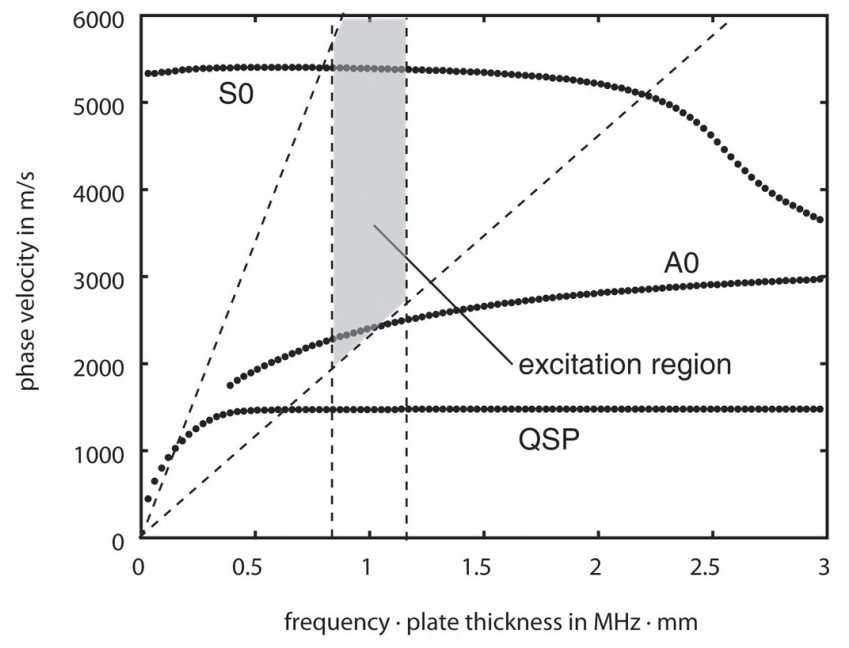

Figure 3. Dispersion diagram of a glass plate in contact with a liquid half-space together with the excitation line and the excitation region of the single-phase transducer. (glass: $v_{1}=5654 \mathrm{~m} \mathrm{~s}^{-1}$, $v_{\mathrm{s}}=3391 \mathrm{~m} \mathrm{~s}^{-1}, \quad \rho=2550 \mathrm{~kg} \mathrm{~m}^{-3}$; water: $v_{\mathrm{l}}=1480 \mathrm{~m} \mathrm{~s}^{-1}, \quad v_{\mathrm{s}}=$ $\left.0.0001 \mathrm{~m} \mathrm{~s}^{-1}, \rho=1000 \mathrm{~kg} \mathrm{~m}^{-3}\right)$.

(Fig. 3). The boundaries of this excitation region are defined by the half bandwidth of the Fourier transform of the spatial distribution of the finger structure and the half bandwidth of the Fourier transform of the excitation signal (Fig. 4).

For a plate without liquid contact, the fundamental antisymmetrical and symmetrical Lamb wave modes are excited. Because the symmetrical zero-order Lamb wave mode has nearly no normal displacement component in the frequency thickness region of $1 \mathrm{MHz}$ mm (Rose, 1999; Viktorov, 1967), only the antisymmetrical zero-order Lamb wave mode can be detected by the laser Doppler vibrometer. Figure 5 shows scanning laser Doppler vibrometer images of the Lamb wave excitation on a $1 \mathrm{~mm}$-thick glass plate at $3.71 \mu \mathrm{s}$ (a), $8.4 \mu \mathrm{s}$ (b) and $14.65 \mu \mathrm{s}$ (c) after the starting time of the excitation signal. In the images, a transition from near-field diffraction to far-field diffraction can be observed. The transition length $x_{F}$ between near-field and far-field diffraction can be described by

$x_{F} \approx \frac{W^{2} k}{10 \pi|b|^{\prime}}$, 

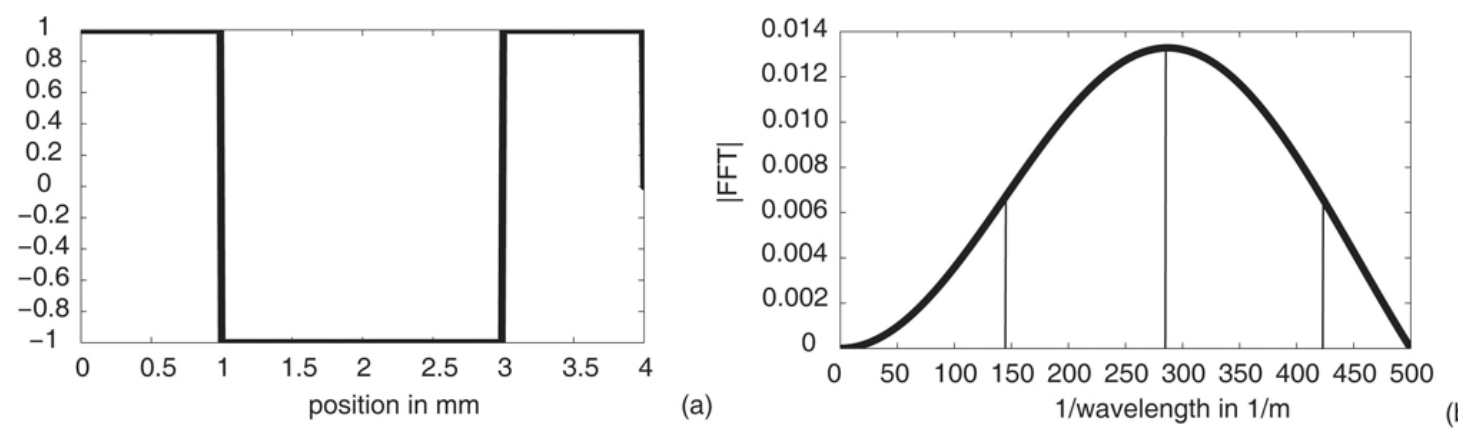

(b)
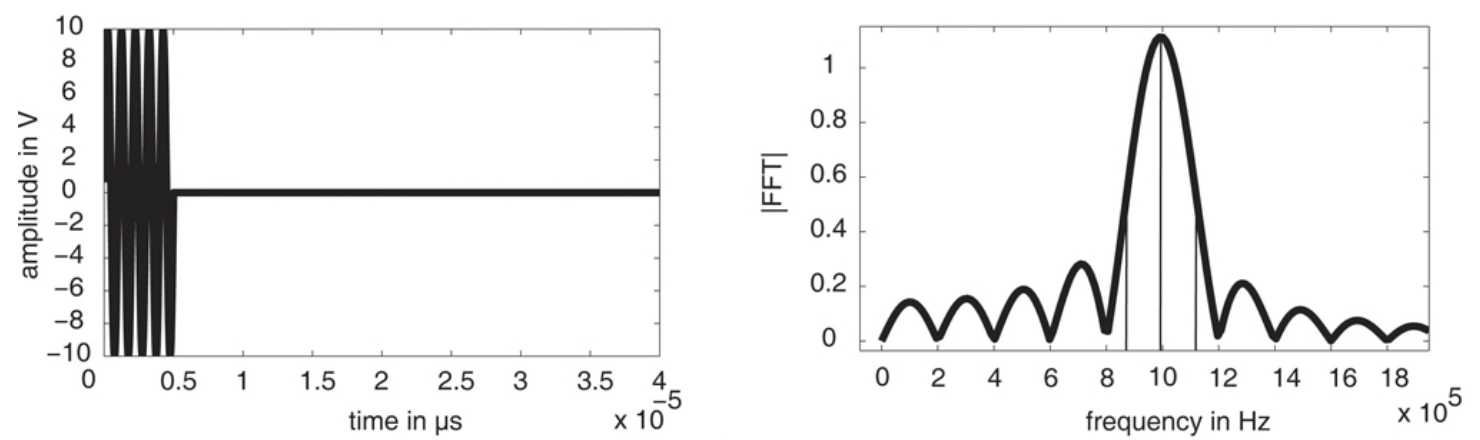

(c)

(d)

Figure 4. (a) Spatial distribution of the two-finger single-phase transducer. (b) Fourier transform of the spatial distribution of the two-finger single-phase transducer with the half bandwidth drawn in. (c) Time signal of the excitation. (d) Fourier transform of the time signal of excitation with the half bandwidth drawn in.

where $W$ is the aperture of the transducer, $k$ is the Lamb wave number and $|b|$ describes the diffraction spreading (Morgan, 2007). In the current case, the glass substrate is isotropic, meaning $b=1$. With the wavelength of the excited antisymmetrical zero-order Lamb wave mode of $3 \mathrm{~mm}$, the thickness of the glass substrate of $1 \mathrm{~mm}$ and the aperture of the transducer of $6 \mathrm{~mm}$, the transition length is

$x_{F} \approx 2.4 \mathrm{~mm}$.

A comparison between the calculated transition length and the scanning laser Doppler vibrometer images proves the calculation that, after this transition length, the emitted wave front is more radial than parallel (Fig. 6).

\section{Mode conversion}

\subsection{Concept of mode conversion}

If the Lamb wave is in contact with liquid, mode conversion occurs. This means that a pressure wave is radiated into the liquid under a characteristic angle, called Lamb angle (Fig. 7). The Lamb angle $\Theta_{\mathrm{L}}$ can be easily calculated by Snell's law:

$\Theta_{\mathrm{L}}=\arcsin \left(\frac{v_{\text {liquid }}}{v_{\text {Lamb }}}\right)$,

where $v_{\text {Lamb }}$ is the Lamb wave velocity and $v_{\text {liquid }}$ is the sound velocity in the liquid.
In order to prove the concept of mode conversion, membrane hydrophone measurements and Schlieren visualization of the radiated sound field are recorded, evaluated and compared with the calculation results.

\subsection{Membrane hydrophone measurements}

The measurement setup for the membrane hydrophone measurements consists of a plate onto which a single-phase transducer with a center frequency of $1 \mathrm{MHz}$ is glued. The transducer is positioned outside the water in such a way that the excited Lamb wave runs into the water (Fig. 8a). By liquid contact, the emission of a pressure wave into the water occurs. A PVDF membrane hydrophone is moved parallel to the plate in all three directions of space by a computercontrolled stepper motor and measures the sound pressure, which is converted into a voltage. Detailed information about the used PVDF membrane hydrophone is given in Wilkens and Molkenstruck (2007). In the measurements, the transducer is operated by a sinusoidal burst of 5 cycles and an amplitude of $70 \mathrm{Vpp}$. Primarily the antisymmetrical zeroorder Lamb wave mode is excited on a glass plate of $1 \mathrm{~mm}$ thickness. The emission profile in the plane orthogonal to the plate and centered orthogonal to the finger structure of the single-phase transducer (Fig. 8b) can be seen in Fig. 9. By using the maximum of every line, a regression line with a standard deviation is calculated, and the slope of the 

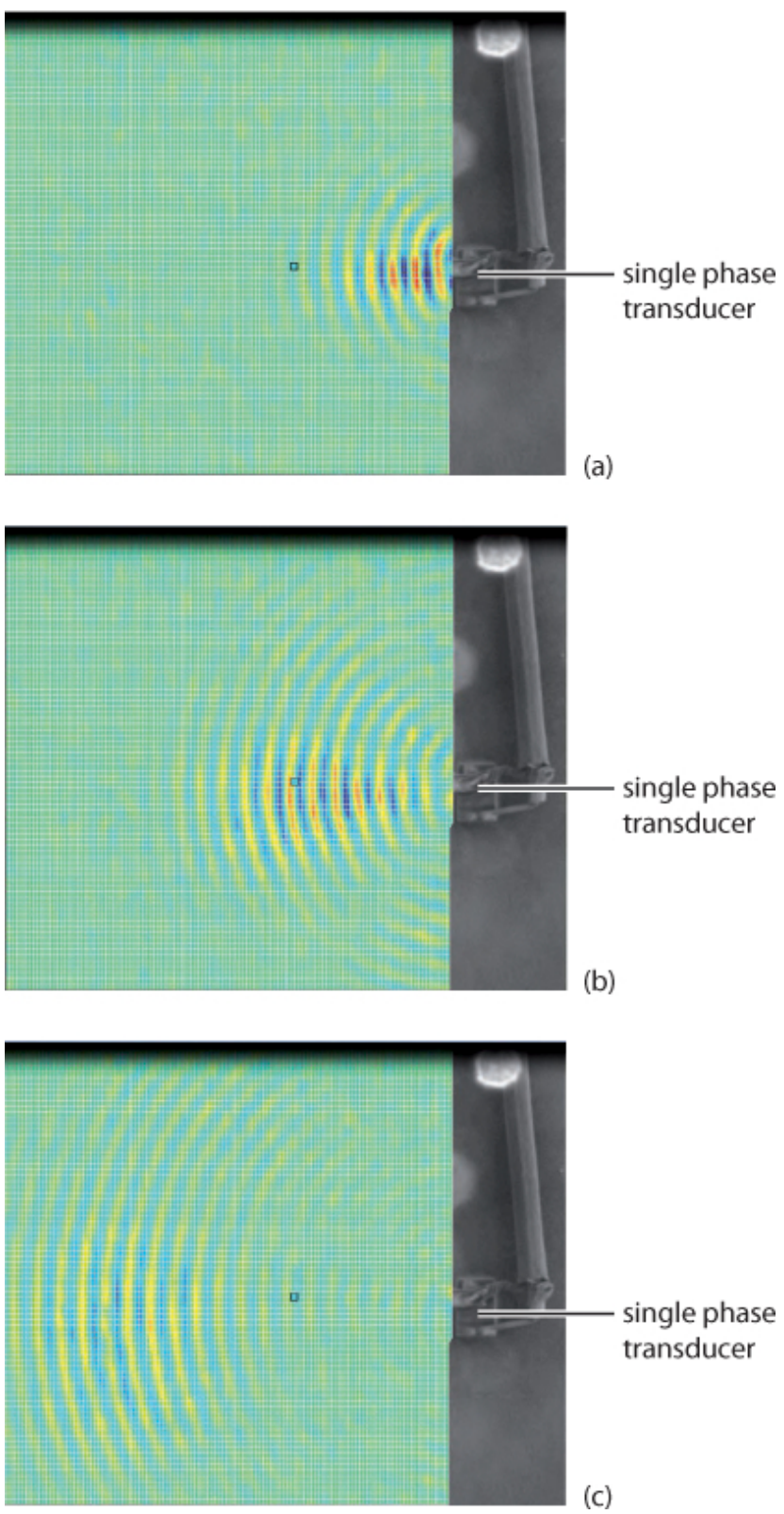

Figure 5. Scanning Laser Doppler vibrometer images of the Lamb wave excitation of a single-phase transducer on a glass plate after (a) $3.71 \mu \mathrm{s}$, (b) $8.4 \mu \mathrm{s}$ and (c) $14.65 \mu \mathrm{s}$ after the excitation signal.

regression line gives the emission angle (Fig. 9). By Snell's law (Eq. 3) and with the knowledge of the sound velocity of $1480 \mathrm{~m} \mathrm{~s}^{-1}$ in water and the Lamb wave phase velocity of the A0 mode of $2210 \mathrm{~m} \mathrm{~s}^{-1}$ for a frequency thickness product of $1 \mathrm{MHz} \times 1 \mathrm{~mm}$ (Fig. 3), the value of the emission angle is evaluated. The comparison between the calculated Lamb angle of

$\Theta_{\mathrm{L}, \text { calculation }}=42^{\circ}$

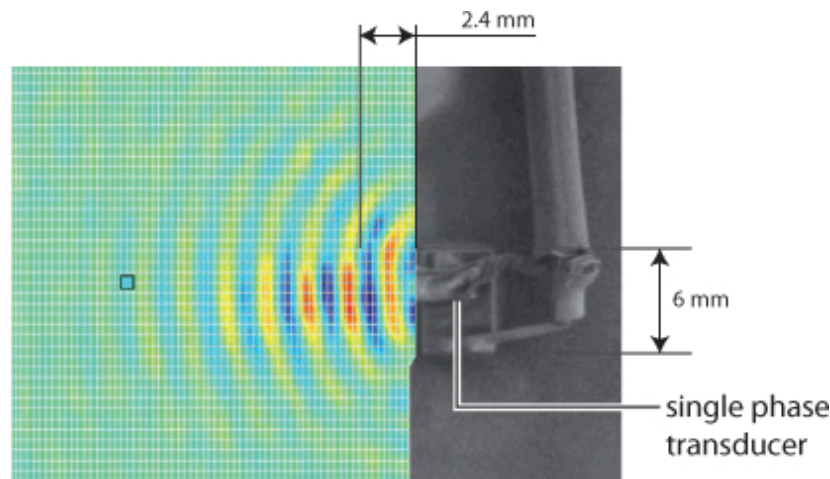

Figure 6. The calculated transition length of diffraction of $2.4 \mathrm{~mm}$ and the aperture of the transducer of $6 \mathrm{~mm}$ are drawn in the scanning laser Doppler image for comparison.

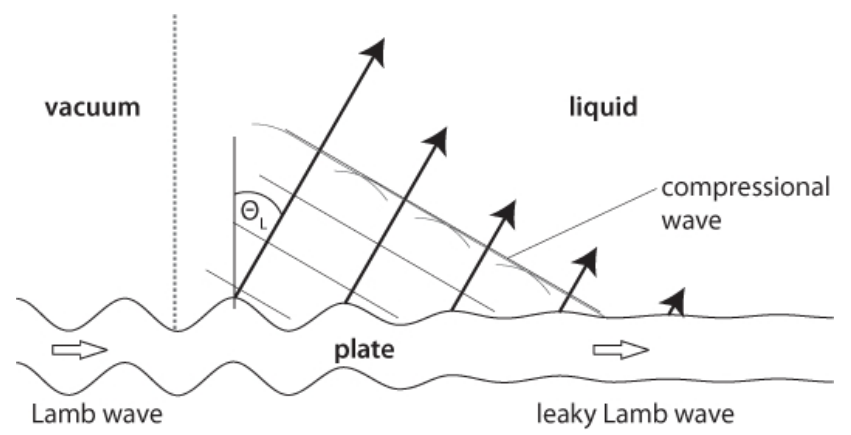

Figure 7. Excitation of a pressure wave into the adjacent liquid by mode conversion of a Lamb wave.

and the measured Lamb angle of

$\Theta_{\mathrm{L}, \text { hydrophone }}=(40.1 \pm 2.4)^{\circ}$.

shows good agreement. Detailed investigations of Lamb wave emission by a single-phase transducer on glass slides with different thicknesses can be found in Schmitt et al. (2012).

\subsection{Schlieren visualization}

The measurement setup for Schlieren visualization consists of a plate onto which a single-phase transducer with a center frequency of $1 \mathrm{MHz}$ is glued. The single-phase transducer is protected from direct contact with the liquid by a housing (Fig. 10a). Lamb waves are excited on the plate by an electrical continuous sinusoidal signal with a peak to peak voltage of $32 \mathrm{~V}$ applied to the transducer. The plate is dipped into the water in such a way that the position of the single-phase transducer is above the liquid level. The waves in the liquid measured by the Schlieren method were emitted from a glass plate of $1.0 \mathrm{~mm}$ thickness. The Schlieren setup consists of a pulsed diode laser, a basin, a digital micromirror device, a CMOS chip and optical components (Fig. 11) (Unverzagt et al., 2010). The propagation of ultrasonic waves produces 

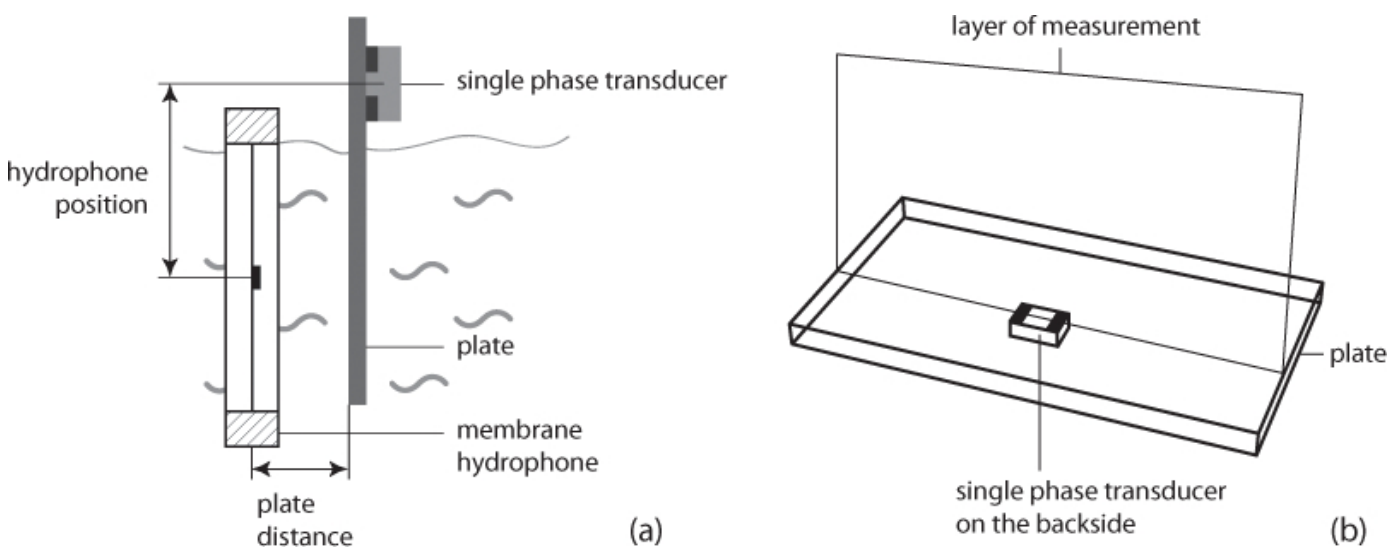

Figure 8. (a) Measurement setup for membrane hydrophone measurements. (b) The emission profile is measured in the plane orthogonal to the plate and centered orthogonal to the finger structure of the single-phase transducer.

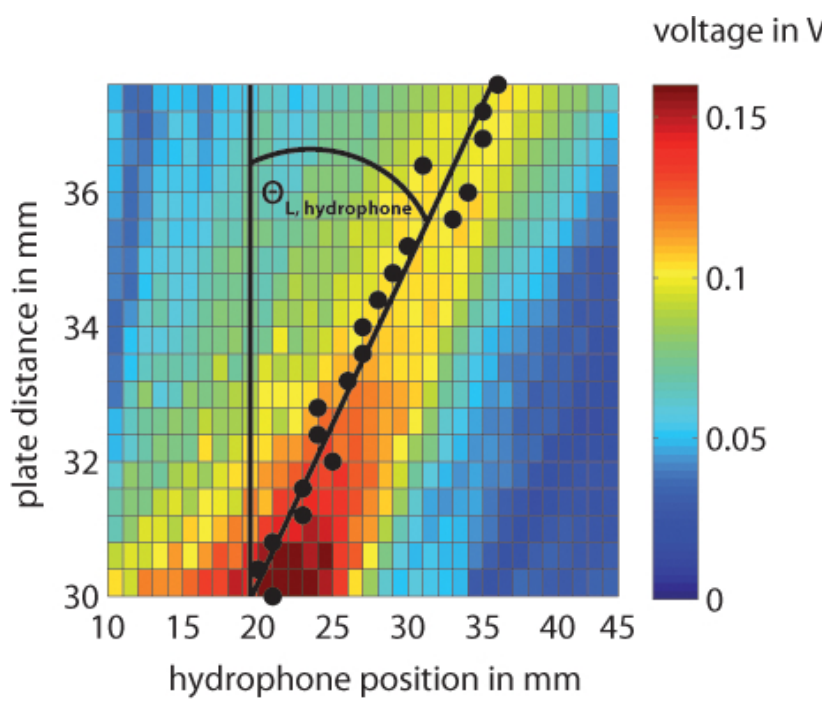

Figure 9. Emission profile of the antisymmetrical zero-order Lamb wave mode on a glass plate of $1 \mathrm{~mm}$ thickness.

density variations within the liquid resulting in a diffraction object for the light. The diffracted light is evaluated to obtain a picture of sound wave propagation within the liquid (Settles, 2001).

The emission of pressure waves into the adjacent water for a glass plate with $1.0 \mathrm{~mm}$ thickness and the position of the single-phase transducer above the liquid level can be seen in Fig. 10b. An emission lobe can be observed in the Schlieren figure, which can be attributed to the emission from the excited A0 Lamb wave mode (Fig. 10b). Moreover, an interference pattern can be seen in the Schlieren figure.

For the comparison with the calculated radiation angle of the A0 Lamb wave mode, the radiation angle of the A0 Lamb wave has been measured. The measured emission angle of the $1 \mathrm{~mm}$-thick glass plate is about

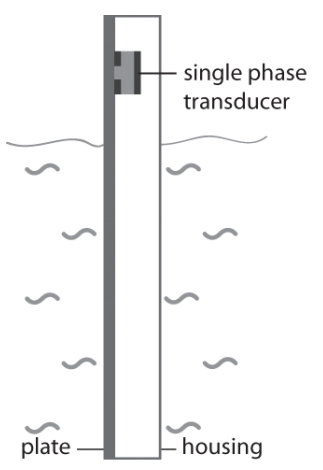

(a)

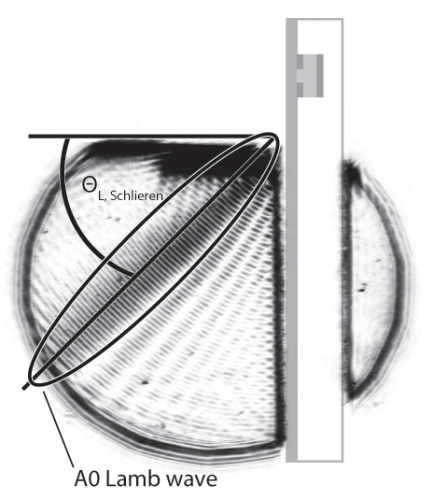

(b)
Figure 10. (a) Measurement setup for Schlieren visualization of Lamb wave emission. (b) Emission profile of acoustic wave excited by a single-phase transducer on a $1 \mathrm{~mm}$-thick glass plate.

$\Theta_{\mathrm{L}, \text { Schlieren }}=45^{\circ}$.

The difference between the measured and calculated emission angles of $42^{\circ}$ (Eq. 4) of the A0 Lamb wave can be attributed to an interference with the emission of the parallel excited symmetrical zero-order Lamb wave mode (Rautenberg et al., 2013).

\section{Inverse mode conversion}

The mode-converted pressure wave in the liquid gets on the opposite side of the tube or container retransformed into a Lamb wave again. In order to prove and visualize this inverse mode conversion process, Schlieren images were made.

The measurement setup consists of two parallel glass slides with a thickness of $1 \mathrm{~mm}$ and a distance between the plates of $12 \mathrm{~mm}$. On one plate, a transmitter is attached on the outer side; on the other plate the emitter is fixed on the 


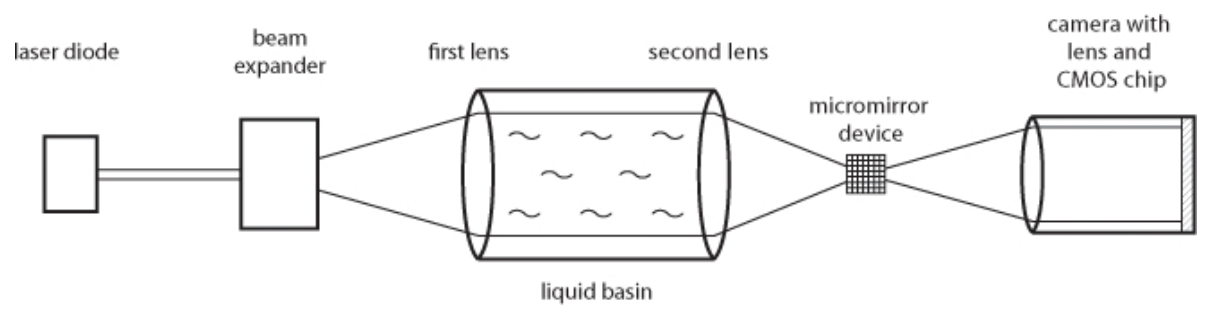

Figure 11. Measurement setup of the optical Schlieren system.
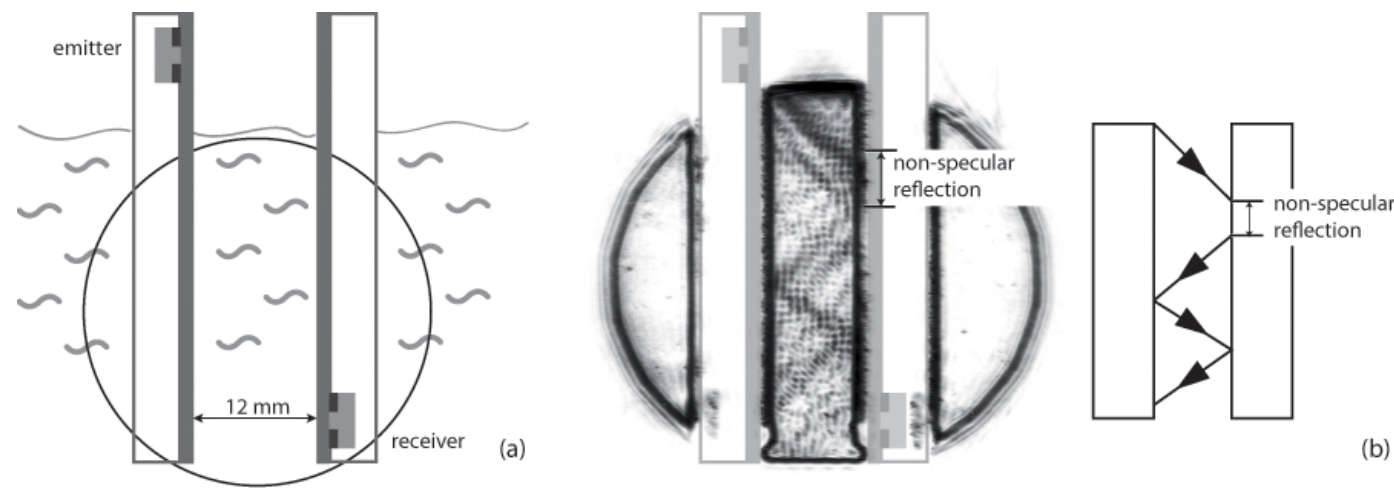

Figure 12. (a) Measurement setup for the Schlieren visualization of the inverse mode conversion process. (b) Schlieren image of the sound wave propagation within the liquid-filled gap of the measurement setup.

outer side too (Fig. 12a). In order to protect the transducers from water, the outer plate sides with the transducers are covered by housings. The setup is dipped into the water in such a way that the transmitter is just above the liquid level (Fig. 12a). The transmitter and the receiver are single-phase transducers with a center frequency of $1 \mathrm{MHz}$, which are designed to excite dominantly the antisymmetrical zero-order Lamb wave mode on thin plates. The transmitter is excited by a continuous sinusoidal signal with a peak to peak voltage of $32 \mathrm{~V}$.

Because the transmitter is above the liquid level, the excited Lamb wave enters into the liquid, and mode conversion occurs therein. This means that an inhomogeneous pressure wave is excited into the liquid with a characteristic Lamb angle. The pressure wave passes the liquid and is reflected back into the liquid again by the second glass slide (Fig. 12b). This process of passing and reflection occurs a few times within the liquid-filled gap between the two plates. In the Schlieren image, the sound propagation way through the liquid can be described by a zigzag pathway (Fig. 12b). Therefore, the parallel plate arrangement behaves like a bulk acoustic waveguide (Rautenberg et al., 2012). In the case of burst excitation, the receiver signal consists of several well-distinguished wave groups, which are evaluated for transmission time and amplitude measurements (Lindner et al., 2006).

Detailed inspection of the first reflection on the right plate reveals a lateral displacement of the outgoing reflected pressure wave (Fig. 12b). This lateral displacement, in the lit- erature called non-specular reflection, corresponds to the Schoch phenomenon and is attributed to Lamb wave excitation and subsequent mode conversion (Schoch, 1952; Pitts et al., 1977). At other places of reflection, no such displacement can be seen. A possible explanation of this phenomenon could be provided by the assumption that, besides the sound waves within the liquid, Lamb waves are propagating on both plates and thus the plates may not be considered to be undisturbed except for the first reflection at the receiver plate.

Further investigations on sound wave propagation within a container structure can be found in Schmitt et al. (2013).

\section{Theoretical considerations}

In order to investigate the effect of coatings on the mode conversion process of a leaky Lamb wave, the global matrix method is utilized. With the global matrix method the dispersion diagram and the emission diagram of Lamb waves in multilayer with contact to liquid half-spaces can be calculated (Lowe, 1995). The extensive equations within the global matrix of a two-layer system with contact to one liquid half-space can be found in Schmitt (2012). As inputs for the global matrix equations, the acoustic material parameters (density, Poisson ratio and Young's modulus or the longitudinal and transversal sound velocities respectively), the thicknesses of both layers (tube/container and coating) and the acoustic material parameters of the liquid half-space must be defined. As outputs the dispersion diagram and the emission 


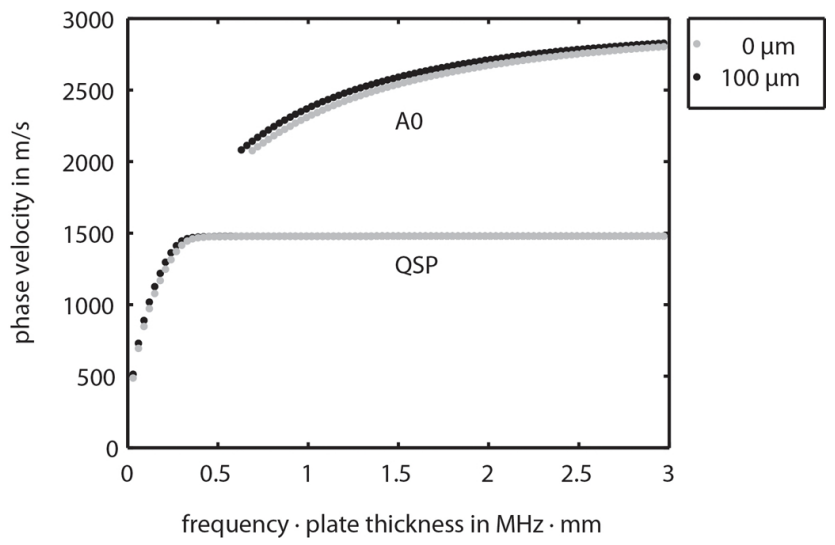

Figure 13. Dispersion diagram of a steel plate $\left(v_{1}=5707 \mathrm{~m} \mathrm{~s}^{-1}\right.$, $\left.v_{\mathrm{s}}=3155 \mathrm{~m} \mathrm{~s}^{-1}, \rho=7850 \mathrm{~kg} \mathrm{~m}^{-3}, d=1 \mathrm{~mm}\right)$ with and without a nickel coating $\left(v_{\mathrm{l}}=5793 \mathrm{~m} \mathrm{~s}^{-1}, v_{\mathrm{s}}=3202 \mathrm{~m} \mathrm{~s}^{-1}, \rho=8000 \mathrm{~kg} \mathrm{~m}^{-3}\right)$ of $100 \mu \mathrm{m}$ thickness in contact with a water half-space $\left(v_{1}=\right.$ $1480 \mathrm{~m} \mathrm{~s}^{-1}, \rho=1000 \mathrm{~kg} \mathrm{~m}^{-3}$ ).

diagram of the two-layer system with contact to one liquid half-space are plotted. For example, in Figs. 13 and 14 the dispersion diagram and the emission diagram of a steel plate with and without a layer of $100 \mu \mathrm{m}$ nickel in contact with one water half-space is illustrated.

The coating of $100 \mu \mathrm{m}$ nickel on the steel plate results in an increase of the phase velocity of the A0 leaky Lamb wave mode (Fig. 13). By Eq. (3), an increase of the phase velocity of the A0 leaky Lamb wave mode leads to a decrease of the Lamb angle. Depending on the position of receiver 1 (Fig. 1), the measured transmission time can increase or decrease. For the emission diagram the nickel coating results in a decrease of the emission coefficient (Fig. 14), which means that the amplitude of the measured acoustic wave on receiver 1 (Fig. 1) will be reduced. For other substrate-coating combinations, individual dispersions and emission diagrams must be calculated.

These theoretical considerations demonstrate the impact of a coating on the measured transmission times and amplitudes. Due to ideal assumptions in the theory of the global matrix method, such as ideal adhesion condition on the interface of the two layers or well-known material parameters, it is hard to compare theoretical with experimental results in an absolute way. As proof of the concept of coating detection within liquid-filled tubes or containers by mode conversion of leaky Lamb waves, measurement results of adhesive tape layers are summarized in the next chapter.

\section{Measurements}

\subsection{Tube}

For the detection of coatings inside a liquid-filled tube, a plastic tube from a medical machine consisting of PES GF20 is modified by adding three single-phase transducers

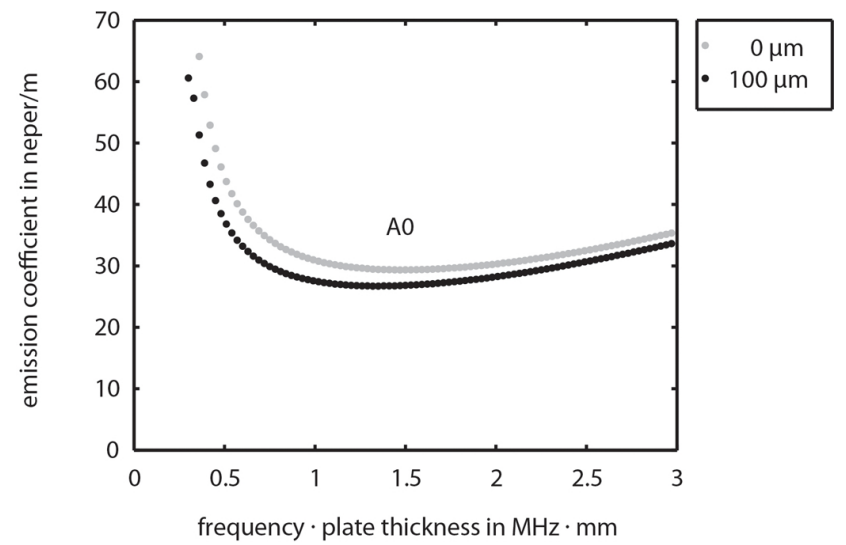

Figure 14. Emission diagram of a steel plate $\left(v_{1}=5707 \mathrm{~m} \mathrm{~s}^{-1}\right.$, $\left.v_{\mathrm{s}}=3155 \mathrm{~m} \mathrm{~s}^{-1}, \rho=7850 \mathrm{~kg} \mathrm{~m}^{-3}, d=1 \mathrm{~mm}\right)$ with and without a nickel coating $\left(v_{\mathrm{l}}=5793 \mathrm{~m} \mathrm{~s}^{-1}, v_{\mathrm{s}}=3202 \mathrm{~m} \mathrm{~s}^{-1}, \rho=8000 \mathrm{~kg} \mathrm{~m}^{-3}\right)$ of $100 \mu \mathrm{m}$ thickness in contact with a water half-space $\left(v_{1}=\right.$ $1480 \mathrm{~m} \mathrm{~s}^{-1}, \rho=1000 \mathrm{~kg} \mathrm{~m}^{-3}$ ).

(Fig. 15a). The single-phase transducers are attached to the outer side of the tube in such a way that a delay line is built up on one side by emitter and receiver 2 . Receiver 1 , which is used for the measurement of the signal propagating inside the liquid, is on the same level as receiver 2 (Fig. 15b). Successively increasing numbers of adhesive tape layers are glued onto the emitter side (Fig. 15b). This results in a shift of the transmission times and the amplitudes of both measured acoustic wave groups on the emitter wall and through the liquid (Fig. 16). The transmission time is measured by a defined zero crossing of the wave group, and the amplitude is evaluated by peak-to-peak measurement of the receiver wave group. The changes of the transmission times and the amplitudes of the signals on the emitter side and through the water versus the thickness of the adhesive tape are depicted in Fig. 17. The increase of the transmission time (Fig. 17b) and the decrease of the amplitude (Fig. 17d) with increasing tape thickness for the receiver signal on the emitter side are primarily caused by a mass effect. The decrease of the transmission time (Fig. 17a) of the acoustic signal through the liquid can be explained by an increase in the Lamb angle. Mass effect on the emitter wall results in a decrease in the phase velocity (Fig. 17b) and therefore in an increase of the Lamb angle (Eq. 3); thus the propagation distance through the water increases too. The decrease in the amplitude of the acoustic signal through the liquid (Fig. 17c) is caused by the hindrance of the emission of pressure wave by mode conversion on the emitter side through the coating and by the decrease of the propagation length of the Lamb wave on the emitter wall, which is caused by an increase of the Lamb angle. Further measurement results of correction fluid and adhesive tape layers in a plastic tube can be found in Lindner et al. (2009). 


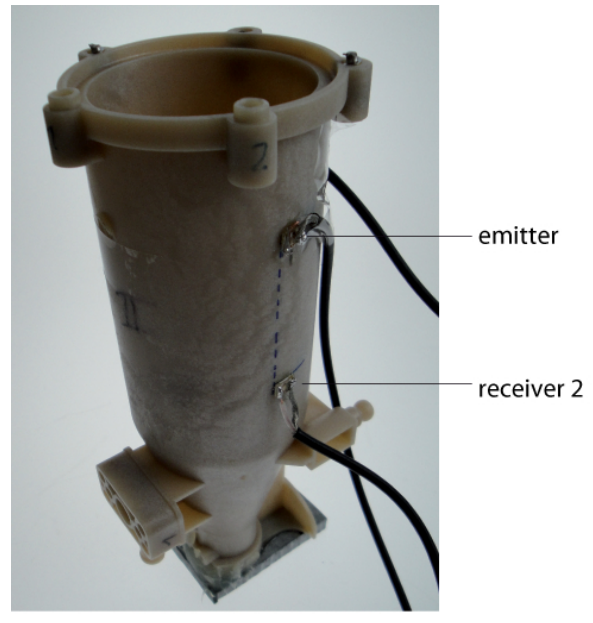

(a)

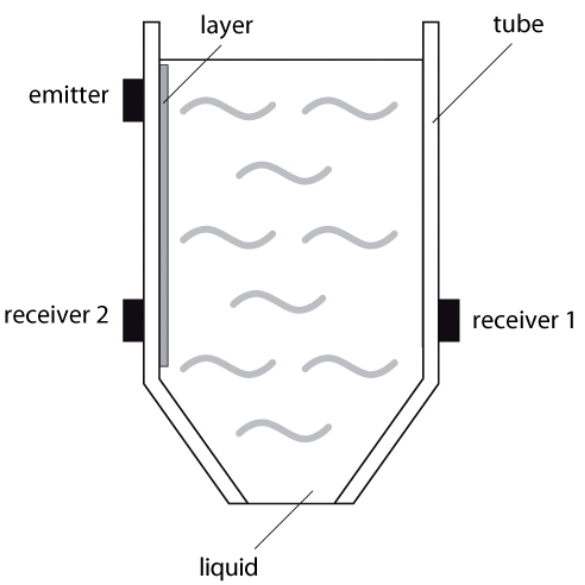

(b)

Figure 15. (a) PES GF 20 tube attached with single-phase transducers. (b) The measurement setup for coating detection.

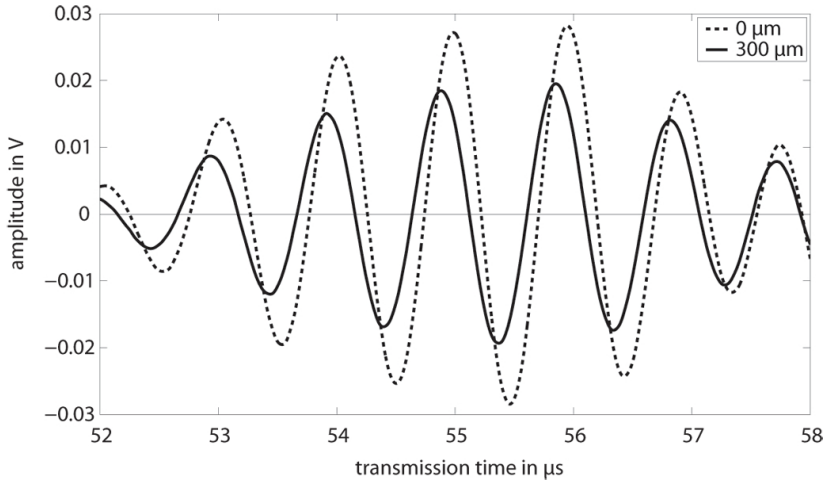

Figure 16. The acoustic receiver signal of receiver 1 through the liquid-filled tube without and with a $300 \mu \mathrm{m}$ coating of adhesive tape on the emitter side.

\subsection{Container}

For the detection of coatings in a container structure, a measurement setup consisting of stainless steel was manufactured (Fig. 18a). It is built up by two parallel steel plates with a distance of $7 \mathrm{~mm}$. Between those plates, water is filled. On the emitter plate, a delay line configuration with an emitter and receiver 2 is positioned, and on the opposite plate receiver 1 is positioned (Fig. 18b). Adhesive tape with the known thickness of $50 \mu \mathrm{m}$ per stripe is glued onto the emitter wall (Fig. 18b). The changes in the measured transmission times and amplitudes of the two receivers are evaluated (Fig. 19). For receiver 2 on the delay line, an increase in the transmission time (Fig. 19b) can be measured; for the amplitude, first an increase until $100 \mu \mathrm{m}$ and then a decrease can be observed (Fig. 19d). The increase in the transmission time is attributed to an increase of mass by the adhesive tape. The increase in the amplitude can be explained by a preven- tion of the mode conversion process by the addition layer on top of the plate in the low thickness range and the decrease in the amplitude by the dominance of the mass effect in the high thickness range. For the signal through the liquid, an increase in the transmission time (Fig. 19a) and a decrease in amplitude (Fig. 19c) occurs. This can be explained by a higher Lamb angle with increasing coating thickness and an extension of the propagation length through the liquid. Further measurement results of correction fluid and adhesive tape layers on glass plates in an acoustic waveguide configuration can be found in Lindner et al. (2009).

\section{Sensitivity and technical realization}

For coating detection by Lamb wave, the transmission time measurements should be evaluated, because the amplitude measurements are not unique and the standard derivations for time measurements are smaller than for amplitude measurements (Fig. 19d). The transmission times of sound waves through the liquid strongly depends on the temperature and the mixture of the fluid. Former experiments demonstrate changes of the transmission time through the liquid of about $50 \mathrm{~ns}^{\circ} \mathrm{C}^{-1}$ in a similar measurement arrangement. The Lamb wave on the emitter wall shows only an increase of about $1 \mathrm{~ns}{ }^{\circ} \mathrm{C}^{-1}$ (Friedrich et al., 2011). Moreover, the influence of the liquid mixture on the transmission time of the Lamb wave is in the low ns range, whereas the transmission time of the Lamb wave through the liquid is in the $\mu$ s range (Friedrich et al., 2011). Compared to the coating effects on the transmission time of around $100 \mathrm{~ns} / 200 \mu \mathrm{m}$ on the emitter side (Fig. 19b) and around $90 \mathrm{~ns} / 200 \mu \mathrm{m}$ through the liquid (Fig. 19a), the temperature and the mixture effect on the acoustic signal have the same magnitude. Thus, for coating detection the transmission time of the residual Lamb wave on the emitter wall should be evaluated, because the influence 

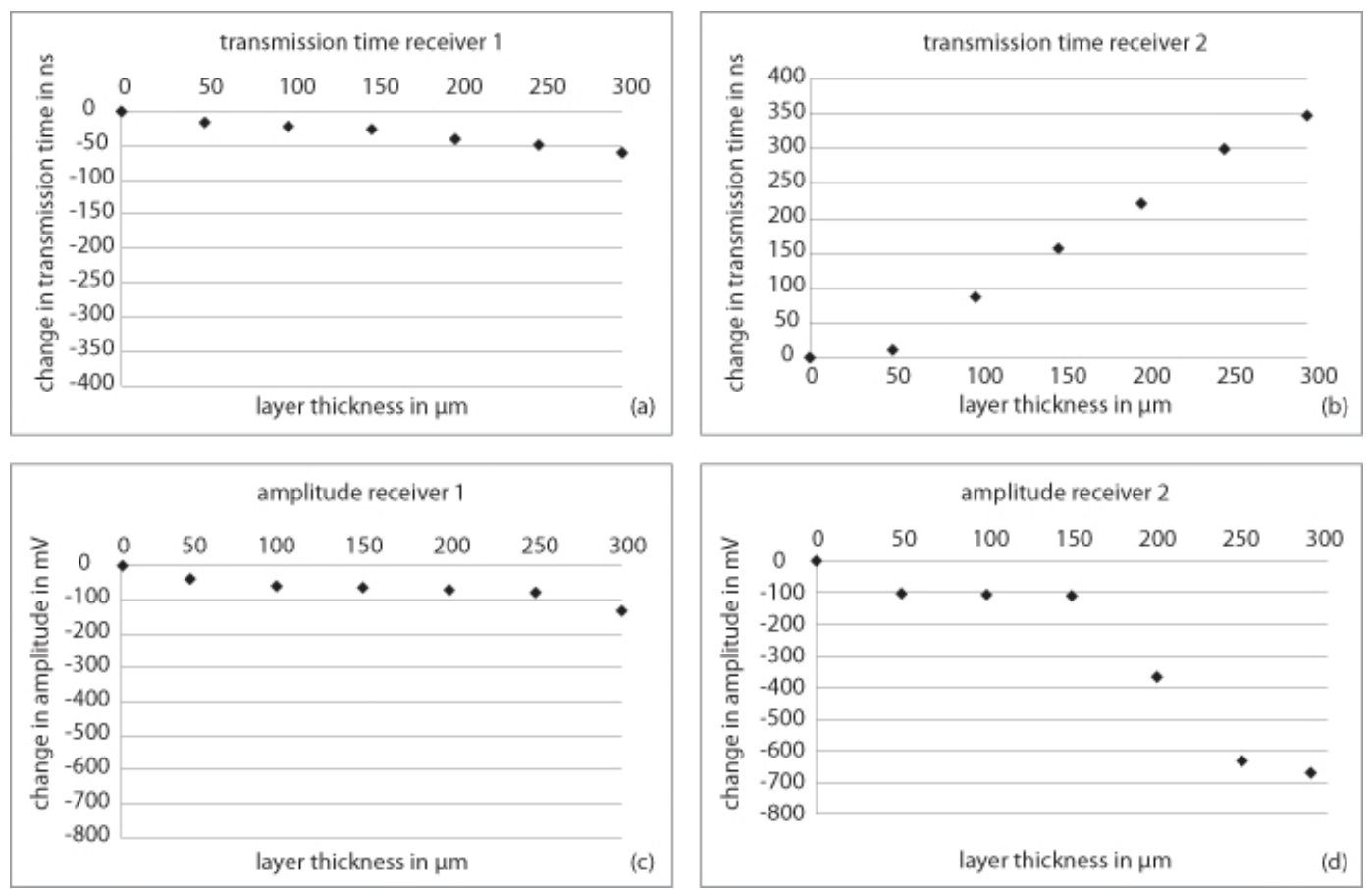

Figure 17. Measurement results of adhesive tape of different thicknesses in the tube for the changes of the transmission times and amplitudes of the acoustic signals on the emitter wall and through the liquid. The standard deviation is too small to be drawn in.

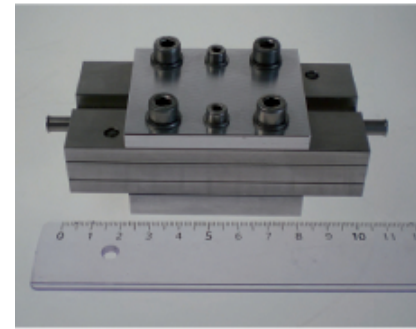

(a)

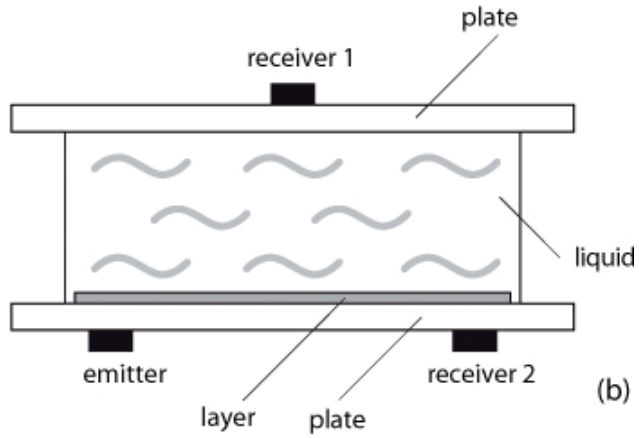

Figure 18. (a) Measurement setup for the container structure consisting of stainless steel. (b) The measurement setup consisting of two parallel plates onto which one emitter and two receivers are attached.

of temperature and liquid mixture is much smaller than the measurement effect in presence of the coating.

Figs. 15a and 18a show measurement setups for labor usage. For a technical realization the transducers must be enclosed in special housings, which can be attached by clamping arrangements and a coupling medium or by gluing to the tube or container. A similar acoustic sensor device has been developed for lubrication surveillance in bearings by leaky Lamb waves and will be brought onto the market (BestSens AG, 2013).

\section{Summary and conclusion}

In this paper, a new measurement concept of coating detection within liquid-filled tubes and containers by the utilization of leaky Lamb waves has been introduced. Scanning laser Doppler vibrometer imaging proves the Lamb wave excitation by the single-phase transducer. Membrane hydrophone measurements and Schlieren measurements confirm the mode conversion process. Schlieren images show the inverse mode conversion of the pressure wave to a Lamb wave on the opposite side of the measurement arrangement. Theoretical considerations demonstrate the dependency of coating on the phase velocity and the emission coefficient of the leaky Lamb wave. Measurement results of coatings 

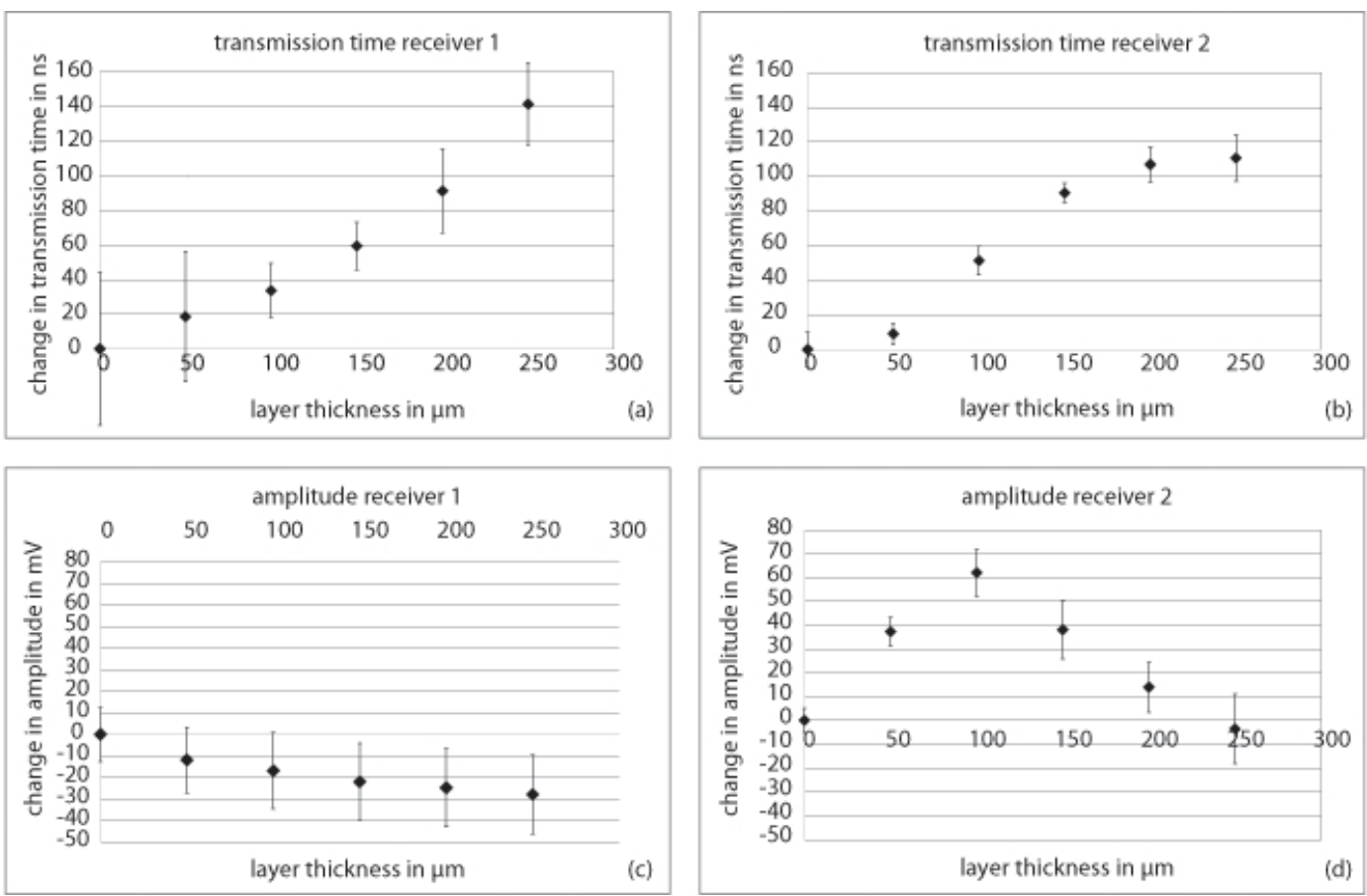

Figure 19. Measurement results for the changes of the transmission times and of the amplitudes on the two receivers for increasing adhesive tape thickness in the stainless steel container.

inside a tube and a container arrangement show a clear dependency on coating thickness for the measured transmission times and amplitudes. For technical realization, the transmission time of the residual Lamb wave is suggested, because of the minor cross sensitivity to temperature and liquid mixture.

The new measurement method of coating detection within liquid-filled tubes and containers by mode conversion of leaky Lamb waves has the huge advantage of a lower detection limit compared to traditional ultrasonic methods such as transmission technique or pulse-echo technique. In this paper it has been demonstrated that the detection range for coating detection by leaky Lamb wave is from 50 to $250 \mu \mathrm{m}$. For traditional ultrasonic transmission technique it is from 500 to $6000 \mu \mathrm{m}$ (Withers, 1996), and for pulse-echo technique it is from 100 to $500 \mu \mathrm{m}$ (Withers, 1996). Moreover, the detection of coating by leaky Lamb wave is non-invasive. This means that the boundary of sterility is not breached, since the single-phase transducers are attached on the outer side of the tube or container wall. Other sensors with such a low detection range, such as fiber optical sensors, electrochemical sensors, pressure sensors or heat sensors, have to be positioned inside the tube or container, causing problems with sterility and cleanability. In the future more work on modeling, especially adhesion conditions and material parameters, and further measurements with different tube or container wall materials and coatings ought to be done, to get calibration models and curves for technical realization.
Acknowledgements. The authors gratefully acknowledge the support obtained from the research teams of the Institute of Sensor and Actuator Technology of Coburg University, the Measurement Engineering Group of Paderborn University and the Microacoustic Group of Freiburg University. The Institute of Sensor and Actuator Technology is supported by European Union within the program "EFRE" (project "Sensolutions").

Edited by: R. Maeda

Reviewed by: two anonymous referees

\section{References}

Balantine, D. S., White, R. M., Martin, S. J., Ricco, A. J., Zellers, E. T., Frye, G. C., and Wohltjen, H.: Acoustic Wave Sensors Theory, Design, and Physico-Chemical Applications, Academic Press, San Diego, 1997.

BestSense AG: Bearing Surveillance \& Test, http://www.bestsens. de/ (last access: 18 December 2012), 2013.

Calabrese, G. S., Wohltjen, H., and Roy, M. K.: Surface acoustic wave devices as chemical sensors in liquids. Evidence disputing the importance of Rayleigh wave propagation, Anal. Chem., 59, 833-837, 1987.

Flemming, H. C.: Role and levels of real-time monitoring for successful anti-fouling strategies - an overview, Water Sci. Technol., 47, 1-8, 2003.

Friedrich, D., Kaul, C., Lindner, G., Faustmann, H., and Münch, M.: Acoustic On-Line Monitoring of Chemical Reactions in Liquids with Integrated Temperature Compensation, Sens. Lett., 9, 1-3, 2011. 
Janknecht, P. and Melo, L. F.: Online biofilm monitoring, Reviews in Environmental Science and Bio/Technology, 2, 269283, 2003.

Länge, K., Rapp, B. E., and Rapp, M.: Surface acoustic wave biosensors: a review, Anal. Bioanal. Chem., 391, 1509-1519, 2008.

Lindner, G.: Sensors and actuators based on surface acoustic waves propagating along solid-liquid interfaces, J. Phys. D. Appl. Phys., 41, 123002, doi:10.1088/0022-3727/41/12/123002, 2008.

Lindner, G., Faustmann, H., Frankenberger, S., Plaum, K., Rothballer, S., and Unterburger, M.: A Versatile Acoustic Waveguide Sensor for Liquids Based on Multiple Mode Conversion at SolidLiquid Interfaces, Proc. IEEE Ultrasonics Symposium, 11811184, 2006.

Lindner, G., Schmitt, M., Schmidt, K., Faustmann, H., Krempel, S., and Schubert, J.: Detection of coatings and measurement of coating thickness on technical substrates using surface acoustic waves in a waveguide configuration, Proc. Sensor + Test Conference SENSOR, 35-40, 2009.

Lohr, K. R. and Rose, J. L.: Ultrasonic guided wave and acoustic impact methods for pipe fouling detection, J. Food Eng., 56, 315324, 2003.

Lowe, M. J. S.: Matrix Techniques for Modeling Ultrasonic Waves in Multilayered Media, IEEE Trans. Ultrason. Ferroelectr. Freq. Control, 42, 525-542, 1995.

Monkhouse, R. S. C., Wilcox, P. D., and Cawley, P.: Flexible interdigital PVDF transducers for the generation of Lamb waves in structures, Ultrasonics, 35, 489-498, 1997.

Morgan, D.: Surface Acoustic Wave Filters, Elsevier, Amsterdam, 2007.

Pitts, L. E., Plona, T. J., and Mayer, W. G.: Theory of Nonspecular Reflection Effects for an Ultrasonic Beam Incident on a Solid Plate in a Liquid, IEEE Trans. Sonics Ultrason., SU-24, 101109, 1977.

Rautenberg, J., Bause, F., and Henning, B.: Guided Acoustic Waves for Liquid Property Measurement (in German), Tech. Mess., 79, 135-142, 2012.
Rautenberg, J., Olfert, S., Bause, F., and Henning, B.: Validation of analytically modeled Leaky Lamb radiation using Schlieren photography, Proc. IEEE Ultrasonics Symposium 2012, in press, 2013.

Rose, J. L.: Ultrasonic Waves in Solid Media, Cambridge University Press, Cambridge, 1999.

Schmitt, M.: Detection of deposition within liquid-filled tubes and containers by mode conversion of Lamb waves (in German), Ph.D. thesis, University Freiburg, http://www.freidok. uni-freiburg.de/volltexte/8820/, 2012.

Schmitt, M., Tietze, S., Liang, W., and Lindner, G.: Measurement of the Emission of Lamb Waves by a PVDF Membrane Hydrophone, AIP Conf. Proc., 1433, 133-136, 2012.

Schmitt, M., Olfert, S., Rautenberg, J., Lindner, G., Henning, B., and Reindl, L. M.: Multi Reflection of Lamb Wave Emission in an Acoustic Waveguide Sensor, Sensors, 13, 2777-2785, 2013.

Schoch, A.: Lateral displacement of total reflected ultrasound waves, Acoustica, 2, 18-19, 1952 (in German).

Settles, G. S.: Schlieren and Shadowgraph Techniques, Springer, Berlin, 2001.

Thompson, M. and Stone, D. C.: Surface-Launched Acoustic Wave Sensor of Chemical Analysis, John Wiley \& Sons, Inc., New York, 1997.

Unverzagt, C., Olfert, S., and Henning, B.: A new method of spatial filtering for Schlieren visualization of ultrasound wave fields, Phys. Procedia, 3, 935-942, 2010.

Viktorov, I. A.: Rayleigh and Lamb Waves, physical theory and applications, Academic Press, New York, 1967.

White, R. M.: Surface Elastic Waves, Proceedings of the IEEE, 58, 1238-1276, 1970.

Wilkens, V. and Molkenstruck, W.: Broadband PVDF Membrane Hydrophone for Comparisons of Hydrophone Calibration Methods up to $140 \mathrm{MHz}$, IEEE Trans. Ultrason. Ferroelectr. Freq. Control, 54, 1784-1791, 2007.

Withers, P. M.: Ultrasonic, acoustic and optical techniques for the non-invasive detection of fouling in food processing equipment, Trends Food Sci. Tech., 7, 293-298, 1996. 